\title{
Caracterização das exigências nutricionais de mudas de Pfaffia glomerata em Argissolo Vermelho distrófico arênico pela técnica do nutriente faltante
}

\author{
Characterization of the nutritional demands of young plants of Pfaffia glomerata in a Paleudalf soil by \\ means of the missing nutrient technique
}

\author{
Etiane Caldeira Skrebsky ${ }^{\mathrm{I}}$ Fernando Teixeira Nicoloso ${ }^{\mathrm{II}}$ Joseila Maldaner $^{\mathrm{I}}$ Renata Rauber $^{\mathrm{III}}$ \\ Gabriel Y Castro ${ }^{\mathrm{IV}}$ Gládis de Oliveira Jucoski ${ }^{\mathrm{I}}$ Danilo Rheinheimer dos Santos ${ }^{\mathrm{V}}$
}

RESUMO

Este trabalho teve por objetivo caracterizar as exigências nutricionais de mudas de ginseng brasileiro (Pfaffia glomerata (Spreng.) Pedersen) em Argissolo Vermelho distrófico arênico pela técnica do nutriente faltante. Plantas de $\boldsymbol{P}$. glomerata produzidas in vitro e aclimatizadas ex vitro foram usadas como material inicial para o experimento em casa de vegetação. Aos 10 dias após o transplante para o solo, através de soluções nutritivas, foram realizadas as adubações correspondentes a nove tratamentos: controle (sem adubação), adubação completa (com macro e micronutrientes) e as omissões individuais de $\mathrm{N}, \mathrm{P}, \mathrm{K}, \mathrm{Ca}, \mathrm{Mg}, \mathrm{S}$ e dos micronutrientes ( $\mathrm{Zn}, \mathrm{B}, \mathrm{Cu}, \mathrm{Fe}, \mathrm{Mn}$ e $\mathrm{Mo}$ ). Aos 60 dias de cultivo, avaliaram-se os parâmetros de crescimento das plantas. O número de folhas por planta foi o parâmetro de crescimento mais afetado pela ausência dos nutrientes $N, K, S$ e P na adubação. $O$ efeito da omissão dos nutrientes na adubação foi diferenciado quanto à partição de biomassa entre os órgãos da planta. A biomassa acumulada pela parte aérea obedeceu à seguinte ordem decrescente, em relação à omissão do nutriente: $\mathrm{Mg} \cong$ micronutrientes $>\mathrm{Ca} \cong$ adubação completa $>P>S>K>N \cong$ controle. Já a biomassa radicular obedeceu à seguinte ordem decrescente: $\mathrm{Mg} \cong$ adubação completa $\geq P \geq$ micronutrientes $\geq$ $S \geq \mathrm{Ca}>N>K \cong$ controle. Plantas jovens de P. glomerata cultivadas em Argissolo Vermelho distrófico arênico apresentaram grande redução no crescimento pela ausência de $N, K, S$ e P na adubação. As omissões de $\mathrm{Mg}$ e dos micronutrientes na adubação não foram limitantes para o crescimento das plantas jovens.

Palavras-chave: ginseng brasileiro, nutrição mineral, omissão de nutrientes, adubação mineral, Amaranthaceae.

\begin{abstract}
This study aimed to characterize the nutritional demands of the young plants of Brazilian ginseng (Pfaffia glomerata (Spreng.) Pedersen) in a Paleudalf soil by means of the missing nutrient technique. Plantlets of P. glomerata produced in vitro and acclimatized ex vitro were used as start matter for the glasshouse experiment. Ten days after plant transfer to soil, fertilizations (by means of nutritive solutions) were done corresponding to nine treatments: control (without fertilization), complete fertilization (with macro and micronutrients), and with the omissions of $N, P, K, C a, M g, S$ and micronutrients ( $\mathrm{Zn}, \mathrm{B}, \mathrm{Cu}, \mathrm{Fe}, \mathrm{Mn}$ and $\mathrm{Mo}$ ). At 60 days of cultivation, several growth parameters were evaluated. The number of leaves per plant was the growth parameter most affected by omission of $N, K, S$ and $P$ in the fertilization. The effect of nutrient omission in the fertilization on biomass partitioning between plant organs were differentiated. Accumulated shoot biomass obeied the following decreasing order, in relation with the nutrient omission: $\mathrm{Mg} \cong$ micronutrients $>\mathrm{Ca} \cong$ complete fertilization $>P>S>K>N \cong$ control. While the root biomass obeied the following decreasing order: $M g \cong$ complete fertilization $\geq P \geq$ micronutrients $\geq S \geq$ $\mathrm{Ca}>N>K \cong$ control. Young plants of $P$. glomerata grow in a Paleudalf soil showed greater reduction in growth by omission of $N, K, S$ and $P$ in the fertilization. The omission of $\mathrm{Mg}$ and micronutrients in the fertilization were not limiting to the growth of young plants.
\end{abstract}

Key words: Brazilian ginseng, mineral nutrition, nutrient subtraction, mineral fertilization, Amaranthaceae.

\footnotetext{
IPrograma de Pós-graduação em Agronomia, Centro de Ciências Rurais (CCR), Universidade Federal de Santa Maria (UFSM), 97105-900, Santa Maria, RS, Brasil.

IIDepartamento de Biologia, UFSM, Camobi, 97105-900, Santa Maria, RS, Brasil. E-mail: ftnicoloso@yahoo.com. Autor para correspondência.

IIICurso de Engenharia Florestal, CCR, UFSM, Santa Maria, RS, Brasil.

${ }^{\text {IV }}$ Curso de Agronomia, CCR, UFSM, Santa Maria, RS, Brasil.

vPrograma de Pós-graduação em Ciências do Solo, Departamento de Solos, UFSM, Santa Maria, RS, Brasil.
} 


\section{INTRODUÇÃO}

Pfaffia glomerata (Spreng.) Pedersen, conhecida como uma das espécies de ginseng brasileiro, é considerada uma planta medicinal devido a possuir características adaptógenas e antiestresse (MONTANARI JR., 1999; MONTANARI JR. et al., 1999; MAGALHÃES, 2000). No Brasil, a P. glomerata tem como principal habitat as matas ciliares da Região Centro-Oeste e a bacia do rio Paraná (MAGALHÃES, 2000).

Toneladas de raízes dessa espécie são mensalmente destinadas ao mercado nacional e internacional. Contudo, a conversão dessas plantas em fármacos está limitada pela dificuldade de se obter matéria-prima para suprir a demanda requerida pela indústria farmacêutica. Atualmente, grande parte das plantas é coletada por extrativismo severo e descontrolado (MONTANARI JR., 1999; MONTANARI JR. et al., 1999; MAGALHÃES, 2000). Portanto, o cultivo dessa espécie se faz totalmente urgente, podendo ser, inclusive, uma interessante alternativa de atividade agrícola (MAGALHÃES, 2000).

As exigências nutricionais da $\boldsymbol{P}$. glomerata são pouco conhecidas, mas dados de ensaios preliminares sugerem que poderá haver respostas à aplicação de nutrientes, uma vez que a produtividade foi maior quando cultivada sobre solos argilosos em comparação a solos arenosos (MAGALHÃES, 2000). O conhecimento de limitações físicas e químicas dos solos e das exigências nutricionais e fisiológicas dessa espécie é fundamental à viabilidade econômicoambiental da inclusão dessa planta no sistema de produção agrícola.

O uso da técnica do nutriente faltante diagnose por subtração - é uma alternativa adequada à iniciação do estudo da resposta das culturas aos nutrientes e pode ser conduzida em condições de casa de vegetação com diferentes substratos e formas de reposição dos nutrientes (MALAVOLTA, 1980; RENO et al., 1997; NICOLOSO et al., 1999; VENTURIN et al., 1999; MOREIRA et al.; 2000; ASSIS et al., 2001; BATISTA et al., 2003; MARQUES et al., 2004; VIÉGAS et al., 2004a, b). De acordo com VIÉGAS et al. (2004a), através dessa técnica de diagnose é possível determinar quais são os nutrientes limitantes ao crescimento e ao estado nutricional das plantas em qualquer tipo de solo. Segundo MALAVOLTA (1980), o método serve para avaliar a fertilidade do solo e, ao mesmo tempo, pode fornecer uma idéia semiquantitativa da necessidade de adubos. Utilizando-se dessa técnica de estudo, NICOLOSO et al. (1999) constataram que um Argissolo Vermelho distrófico arênico se apresentou deficiente em N, P, S e K para o máximo crescimento da grápia (Apuleia leiocarpa), assim demonstrando que esse solo apresenta características químicas que facilitam os estudos que visam a identificar os efeitos das omissões de nutrientes na adubação.

As diferentes características do crescimento das espécies vegetais, bem como de suas exigências nutricionais, são fatores que podem explicar os diferentes comportamentos das plantas quanto à utilização de recomendações de calcário e de fertilizantes baseadas em resultados experimentais médios obtidos em distintas condições de solo e ambiente. A necessidade da determinação de níveis críticos de um nutriente para cada espécie vegetal ou grupos de espécies afins fundamenta-se não apenas em suas exigências nutricionais variáveis, mas também nas suas diferentes capacidades de absorção e/ou utilização do nutriente (BARROS \& NOVAIS, 1990; MARSCHNER, 2002).

O presente trabalho teve por objetivo caracterizar as exigências nutricionais de mudas de $\boldsymbol{P}$. glomerata em Argissolo Vermelho distrófico arênico pela técnica do nutriente faltante.

\section{MATERIAL E MÉTODOS}

Plantas de P. glomerata (Spreng.) Pedersen, multiplicadas in vitro de acordo com NICOLOSO et al. (2001a) e aclimatizadas segundo a metodologia de SKREBSKY et al. (2006), foram usadas no ensaio. Segmentos nodais de $1 \mathrm{~cm}$ de comprimento foram inoculados em meio de cultura MS (MURASHIGE \& SKOOG, 1962), suplementado de $30 \mathrm{~g} \mathrm{~L}^{-1}$ de sacarose, $100 \mathrm{mg} \mathrm{L}^{-1}$ de mio-inositol e $6 \mathrm{~g} \mathrm{~L}^{-1}$ de agar. Após a inoculação, os explantes foram cultivados em sala de crescimento com temperatura controlada de $25 \pm 2^{\circ} \mathrm{C}$, fotoperíodo de 16 horas e intensidade luminosa de $35 \mu \mathrm{mol} \mathrm{m} \mathrm{m}^{-2} \mathrm{~s}^{-1}$ através de lâmpadas fluorescentes brancas frias. Aos 25 dias após a inoculação, procedeuse ao início da aclimatização através da abertura dos tubos de cultivo em sala de crescimento, por período de três dias ( $1^{\underline{a}}$ etapa); após, fez-se a transferência das plantas para recipientes de plástico $\left(350 \mathrm{~cm}^{3}\right)$ contendo $250 \mathrm{~cm}^{3}$ de substrato Plantmax ${ }^{\circledast}$ Hortaliças, as quais ficaram 15 dias em sala de crescimento ( 2 a etapa). $\mathrm{Na}$ seqüência, as plantas foram transferidas para um telado de sombrite (malha $50 \%$ de extinção de luz), onde ficaram mais 15 dias ( $3^{a}$ etapa). Essas plantas aclimatizadas foram transplantadas para solo em ambiente de casa de vegetação, localizada no Departamento de Fitotecnia da Universidade Federal de Santa Maria.

O solo utilizado foi coletado do horizonte A (zero a $0,65 \mathrm{~cm}$ de profundidade) de um Argissolo 
Vermelho distrófico arênico ocorrente no município de Santa Maria, RS. Os dados das análises físicas e químicas desse solo após a coleta, destorroamento e peneiramento, bem como na presença dos tratamentos de adubação no momento da finalização do experimento, são apresentados na tabela 1 .

A unidade experimental foi constituída de um vaso, forrado internamente com saco plástico para evitar a perda de água e nutrientes pela drenagem, contendo $3 \mathrm{~kg}$ de terra fina secada ao ar (malha $0,5 \mathrm{~cm}$ ) e uma planta. Os vasos foram submetidos a rodízio periódico para evitar o efeito de localização na casa de vegetação. $\mathrm{O}$ delineamento experimental usado foi de tratamentos inteiramente casualizados, com 15 repetições.

Aos 10 dias após o transplante, foram realizadas as adubações, constituindo os nove tratamentos, como segue: controle (sem adubação), adubação completa (com macro e micronutrientes) e as omissões individuais de $\mathrm{N}, \mathrm{P}, \mathrm{K}, \mathrm{Ca}, \mathrm{Mg}, \mathrm{S}$ e dos micronutrientes ( $\mathrm{Zn}, \mathrm{B}, \mathrm{Cu}, \mathrm{Fe}, \mathrm{Mn}$ e $\mathrm{Mo})$. A adubação completa $\left(\mathrm{mg} \mathrm{kg}^{-1}\right)$ consistiu em: $\mathrm{N}=100 ; \mathrm{P}=100 ; \mathrm{K}=80$; $\mathrm{Ca}=40 ; \mathrm{Mg}=20 ; \mathrm{S}=15 ; \mathrm{Zn}=1 ; \mathrm{B}=0,25 ; \mathrm{Cu}=0,1 ; \mathrm{Fe}=5,0$; $\mathrm{Mn}=0,1 ; \mathrm{Mo}=0,05$. Os tratamentos de adubação com omissão foram fornecidos em concentrações iguais exceto o nutriente subtraído. As fontes de nutrientes foram: $\mathrm{NH}_{4} \mathrm{H}_{2} \mathrm{PO}_{4} ; \mathrm{KH}_{2} \mathrm{PO}_{4} ; \mathrm{NaH}_{2} \mathrm{PO}_{4} ; \mathrm{KCl}$;
$\mathrm{MgSO}_{4} .7 \mathrm{H}_{2} \mathrm{O} ; \mathrm{NaSO}_{4} ; \mathrm{CaCl}_{2} .2 \mathrm{H}_{2} \mathrm{O} ; \mathrm{KNO}_{3}$; $\mathrm{Ca}\left(\mathrm{NO}_{3}\right)_{2} ; 4 \mathrm{H}_{2} \mathrm{O} ; \mathrm{NH}_{4} \mathrm{NO}_{3} ; \mathrm{MgCl}_{2} .6 \mathrm{H}_{2} \mathrm{O} ; \mathrm{ZnSO} .7 \mathrm{H}_{2} \mathrm{O}$; $\mathrm{ZnCl}_{2} ; \mathrm{H}_{3} \mathrm{BO}_{3} ; \mathrm{CuSO}_{4} .5 \mathrm{H}_{2} \mathrm{O} ; \mathrm{FeSO}_{4} .7 \mathrm{H}_{2} \mathrm{O}$-EDTA; $\mathrm{MnCl}_{2} \cdot 4 \mathrm{H}_{2} \mathrm{O}$ e $\mathrm{H}_{2} \mathrm{MoO}_{4} \cdot \mathrm{H}_{2} \mathrm{O}$. As adubações foram adicionadas no solo na forma de soluções nutritivas em doses únicas. As doses utilizadas neste trabalho são semelhantes àquelas utilizadas por NICOLOSO et al. (1999).

Irrigações diárias foram efetuadas com água destilada, procurando-se manter a umidade do solo entre 50 e $60 \%$ da capacidade de campo, por meio de aferições diárias de pesagem.

No final do experimento, aos 60 dias de cultivo, avaliaram-se a altura da maior brotação, o número de folhas, a matéria seca de folhas, do caule, da parte aérea, de raízes e do total da planta, a relação de matéria seca de raízes e da parte aérea, bem como o crescimento relativo em matéria seca das raízes e da parte aérea. Os dados foram submetidos à análise de variância e as médias comparadas pelo teste de Duncan $(\mathrm{P}<0,05)$.

\section{RESULTADOS E DISCUSSÃO}

O número de folhas por planta obedeceu a seguinte ordem decrescente, em relação à omissão do nutriente: adubação completa $\cong$ micronutrientes $\cong \mathrm{Mg}$

Tabela 1 - Resultados das análises químicas e físicas de uma amostra composta (15 repetições) de um Argissolo Vermelho distrófico arênico do horizonte A, em condições de solo natural e após aplicação dos tratamentos de adubação aos 60 dias de cultivo com mudas de Pfaffia glomerata. UFSM, Santa Maria, 2006.

\begin{tabular}{|c|c|c|c|c|c|c|c|c|c|c|}
\hline \multirow{3}{*}{ Parâmetros } & \multirow{3}{*}{$\begin{array}{l}\text { Adubação } \\
\text { completa }\end{array}$} & \multirow{2}{*}{\multicolumn{7}{|c|}{ }} & \multirow{3}{*}{ controle } & \multirow{3}{*}{$\begin{array}{c}\text { Solo } \\
\text { natural }\end{array}$} \\
\hline & & & & & & & & & & \\
\hline & & $\mathrm{N}$ & $\mathrm{P}$ & $\mathrm{K}$ & $\mathrm{Ca}$ & $\mathrm{Mg}$ & $\mathrm{S}$ & Micro & & \\
\hline Argila (\%) ${ }^{(1)}$ & 17 & 19 & 19 & 20 & 18 & 19 & 18 & 19 & 17 & 18 \\
\hline $\mathrm{pH}-\mathrm{H}_{2} \mathrm{O}^{(2)}$ & 5,0 & 4,3 & 4,2 & 4,9 & 5,2 & 5,1 & 4,5 & 5,0 & 4,6 & 4,7 \\
\hline SMP & 6,2 & 6,2 & 6,2 & 6,3 & 6,2 & 6,3 & 6,4 & 6,3 & 6,1 & 6,2 \\
\hline$\%$ M.O. ${ }^{(3)}$ & 1,8 & 1,6 & 1,5 & 1,5 & 1,4 & 1,4 & 1,5 & 1,5 & 1,9 & 1,4 \\
\hline $\mathrm{P}\left(\mathrm{mg} \mathrm{kg}^{-1}\right)^{(4)}$ & 69 & 69 & 4,8 & 69 & 69 & 69 & 69 & 69 & 3,5 & 2,5 \\
\hline $\mathrm{K}\left(\mathrm{mg} \mathrm{dm}^{-3}\right)^{(4)}$ & 48 & 58 & 42 & 20 & 46 & 50 & 56 & 46 & 22 & 22 \\
\hline $\mathrm{Al}\left(\mathrm{cmol}_{\mathrm{c}} \mathrm{dm}^{-3}\right)^{(5)}$ & 0,5 & 0,6 & 1,0 & 0,5 & 0,5 & 0,7 & 0,5 & 0,6 & 1,1 & 1,4 \\
\hline $\mathrm{Ca}\left(\mathrm{cmol}_{\mathrm{c}} \mathrm{dm}^{-3}\right)^{(5)}$ & 2,0 & 1,9 & 1,6 & 2,1 & 1,6 & 1,8 & 2,0 & 1,9 & 1,7 & 1,4 \\
\hline $\mathrm{Mg}\left(\mathrm{cmol}_{\mathrm{c}} \mathrm{dm}^{-3}\right)^{(5)}$ & 0,6 & 0,9 & 0,6 & 0,6 & 0,6 & 0,3 & 0,7 & 0,6 & 0,5 & 0,4 \\
\hline $\mathrm{H}+\mathrm{Al}\left(\mathrm{cmol}_{\mathrm{c}} \mathrm{L}^{-1}\right)^{(6)}$ & 3,0 & 3,0 & 3,0 & 2,7 & 3,0 & 2,7 & 2,5 & 2,7 & 3,3 & 3,0 \\
\hline CTC ef. $\left(\mathrm{cmol}_{\mathrm{c}} \mathrm{L}^{-1}\right)$ & 3,2 & 3,8 & 3,3 & 3,3 & 2,8 & 2,8 & 3,4 & 3,0 & 3,4 & 3,3 \\
\hline CTC pH $7\left(\mathrm{cmol}_{\mathrm{c}} \mathrm{L}^{-1}\right)$ & 5,6 & 6,2 & 5,3 & 5,5 & 5,2 & 4,8 & 5,4 & 5,1 & 5,5 & 4,9 \\
\hline Sat. Al (\%) & 16 & 16 & 30 & 15 & 18 & 25 & 15 & 20 & 33 & 43 \\
\hline Sat. Bases $(\%)$ & 47 & 52 & 44 & 50 & 43 & 43 & 54 & 47 & 41 & 39 \\
\hline $\mathrm{Cu}\left(\mathrm{mg} \mathrm{dm}^{-3}\right)^{(7)}$ & 1,2 & 1,2 & 1,1 & 1,2 & 1,4 & 1,2 & 1,2 & 1,1 & 1,1 & 1,1 \\
\hline $\mathrm{Zn}\left(\mathrm{mg} \mathrm{dm}^{-3}\right)^{(7)}$ & 6,3 & 6,4 & 5,6 & 5,8 & 7,2 & 5,7 & 4,1 & 4,6 & 2,5 & 1,6 \\
\hline $\mathrm{Fe}\left(\mathrm{mg} \mathrm{dm}^{-3}\right)^{(7)}$ & 70 & 73 & 50 & 71 & 75 & 74 & 72 & 48 & 45 & 49 \\
\hline $\operatorname{Mn}\left(\mathrm{mg} \mathrm{dm}^{-3}\right)^{(8)}$ & 20 & 21 & 23 & 17 & 22 & 23 & 22 & 18 & 22 & 21 \\
\hline $\mathrm{S}\left(\mathrm{mg} \mathrm{L}^{-1}\right)^{(9)}$ & 19 & 31 & 18 & 24 & 23 & 24 & 8,9 & 23 & 9,8 & 13 \\
\hline $\mathrm{B}\left(\mathrm{mg} \mathrm{dm}^{-3}\right)^{(10)}$ & 1,3 & 1,2 & 0,5 & 1,3 & 1,5 & 0,9 & 1,4 & 1,1 & 0,9 & 0,9 \\
\hline
\end{tabular}

(1) método do densímetro; (2) relação $1: 1$; (3) oxidação em solução sulfocrômica a quente e determinação por espectofotometria com Cr ${ }^{+3}$; (4) $\mathrm{P}$ e K, extrator $\mathrm{HCl} 0,05 \mathrm{~N}+\mathrm{H}_{2} \mathrm{SO}_{4} 0,025 \mathrm{~N}$; (5) $\mathrm{Ca}, \mathrm{Mg}$ e Al, extrator $\mathrm{KCl} 1 \mathrm{~N}$; (6) $\mathrm{H}+\mathrm{Al}$, extrator $\mathrm{CaOAc} 1 \mathrm{~N}$ a pH = 7; (7) $\mathrm{Cu}, \mathrm{Zn}$ e $\mathrm{Fe}$, extrator $\mathrm{HCl} 0,1 \mathrm{~N} ;(8) \mathrm{Mn}$, extrator $\mathrm{KCl} 1 \mathrm{M} ;(9) \mathrm{S}$, extrator $\mathrm{Ca}\left(\mathrm{H}_{2} \mathrm{PO}_{4}\right) \cdot \mathrm{H}_{2} \mathrm{O} 2,02 \mathrm{~g} \mathrm{~L}^{-1}$; (10) B, extrator água destilada a quente. 
$\cong \mathrm{Ca}>\mathrm{P}>\mathrm{S} \cong \mathrm{K}>\mathrm{N} \cong$ controle (Tabela 2 ). A diferença de crescimento obtida pelos tratamentos de adubação completa em relação ao controle foi aproximadamente de $1.000 \%$.

A altura da maior brotação seguiu a mesma ordem decrescente em relação aos tratamentos de adubação observada para o número de folhas, com exceção do tratamento de omissão de $P$, que proporcionou crescimento igual ao dos tratamentos de adubação completa e omissão de micronutrientes, $\mathrm{Ca}$ e $\mathrm{Mg}$ (Tabela 2). A diferença de crescimento em altura foi de aproximadamente $67 \%$ entre os tratamentos de maior e menor resposta. Levando-se em consideração o padrão de desenvolvimento da $\boldsymbol{P}$. glomerata, com respeito à emissão de muitas brotações durante a fase vegetativa juvenil, fica caracterizado que o efeito das deficiências nutricionais observadas no presente trabalho foi mais pronunciado sobre o número de folhas do que na altura da maior brotação.

Avaliando-se o desempenho de mudas de uma espécie florestal (Apuleia leiocarpa) utilizando o mesmo solo, NICOLOSO et al. (1999) observaram que as omissões de $\mathrm{P}, \mathrm{N}$ e $\mathrm{S}$ foram as mais depressivas no número de folhas e na altura de plantas. Na deficiência de $\mathrm{P}$, os efeitos mais marcantes relatados em outros trabalhos são a diminuição da área foliar (FREDEEN et al., 1989) e do número de folhas (LYNCH et al., 1991). Portanto, os dados obtidos para o crescimento da $\boldsymbol{P}$. glomerata estão de acordo com a baixa disponibilidade de $\mathrm{N}$ e $\mathrm{S}$, decorrentes dos baixos teores de matéria orgânica e da baixa disponibilidade natural de K e P dos solos arenosos ocorrentes no Rio Grande do Sul. Por outro lado, a disponibilidade natural de micronutrientes, $\mathrm{Ca}$ e $\mathrm{Mg}$ foram suficientes para atender à demanda nutricional dessa planta (Tabela 2), como já tem sido observado inclusive para culturas de grãos, como a soja e o milho. Resultados semelhantes a estes foram encontrados para a grápia (Apuleia leiocarpa), em que diversos parâmetros do crescimento das plantas não foram alterados significativamente pela omissão de $\mathrm{Ca}, \mathrm{Mg}$ e micronutrientes na adubação em Argissolo Vermelho distrófico arênico (NICOLOSO et al., 1999).

A biomassa de folhas seguiu a seguinte ordem decrescente, em relação à omissão do nutriente: adubação completa $\cong$ micronutrientes $\cong \mathrm{Ca} \geq \mathrm{Mg} \geq \mathrm{P}>$ $\mathrm{S}>\mathrm{K}>\mathrm{N} \cong$ controle (Tabela 2). Para a biomassa do caule, a omissão de $\mathrm{Ca}$ e a adubação completa mostraram menor produção do que aqueles de omissão de $\mathrm{Mg}$ e micronutrientes. Este fato se refletiu na biomassa acumulada pela parte aérea (Tabela 2), que obedeceu a seguinte ordem decrescente, em relação à omissão do nutriente: $\mathrm{Mg} \cong$ micronutrientes $>\mathrm{Ca} \cong$ adubação completa $\geq \mathrm{P} \geq \mathrm{S}>\mathrm{K}>\mathrm{N} \cong$ controle. Considerando-se que a $\boldsymbol{P}$. glomerata apresenta alta taxa de crescimento durante a fase vegetativa juvenil, é justificado o fato da ausência do nitrogênio na adubação ser aquela que mais drasticamente reduziu a biomassa da parte aérea.

A produção de biomassa radicular obedeceu a seguinte ordem decrescente, em relação à omissão do nutriente: $\mathrm{Mg} \cong$ adubação completa $\geq \mathrm{P} \geq$ micronutrientes $\geq \mathrm{S} \geq \mathrm{Ca}>\mathrm{N}>\mathrm{K} \cong$ controle (Tabela 2). Esse resultado caracteriza que a partição de fotoassimilados entre a parte aérea e raízes foi diferenciada na omissão de $\mathrm{P}$, micronutrientes, $\mathrm{S}, \mathrm{Ca}, \mathrm{N}$ e K. Nesse contexto, a omissão de $\mathrm{P}$ foi aquela que menos reduziu a biomassa radicular, demonstrando que, na deficiência desse nutriente, a planta redireciona a distribuição de fotoassimilados para as raízes, fato relatado para outras espécies vegetais por FÖHSE et al. (1988), e/ou a P. glomerata no início de seu desenvolvimento vegetativo é pouco exigente nesse

Tabela 2 - Número de folhas ( $\mathrm{n}^{\mathrm{o}}$ fol), altura da maior brotação (alt), matéria seca de folhas (msF), matéria seca de caule (msC), matéria seca da parte aérea (msPA), matéria seca de raízes (msR) e matéria seca total da planta (msT) de Pfaffia glomerata aos 60 dias de cultivo em um Argissolo Vermelho distrófico arênico.

\begin{tabular}{|c|c|c|c|c|c|c|c|}
\hline \multirow{2}{*}{ Tratamentos de adubação } & \multicolumn{7}{|c|}{---------------------------------------------'Parâmetros de crescimento--------------------------------------------- } \\
\hline & №-fol & Alt & $\mathrm{msF}$ & $\mathrm{msC}$ & msPA & msR & msT \\
\hline & (un) & $(\mathrm{cm})$ & -------- & 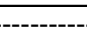 & $\mathrm{g}_{\text {planta }}^{-1}$ & --------- & ------- \\
\hline Completa & $334,1 \mathrm{a}^{*}$ & $85,9 \mathrm{a}$ & $11,9 \mathrm{a}$ & $24,1 \mathrm{~b}$ & $36,0 \mathrm{~b}$ & $9,7 \mathrm{ab}$ & $45,7 \mathrm{bc}$ \\
\hline Omissão de N & $35,7 \mathrm{~d}$ & $56,1 \mathrm{c}$ & $5,5 \mathrm{e}$ & $7,9 \mathrm{f}$ & $13,4 \mathrm{f}$ & $7,0 \mathrm{f}$ & $20,4 \mathrm{~g}$ \\
\hline Omissão de P & $267,2 \mathrm{~b}$ & $91,1 \mathrm{a}$ & $11,2 \mathrm{~b}$ & $21,1 \mathrm{c}$ & $32,4 \mathrm{c}$ & $9,1 \mathrm{bc}$ & $41,6 \mathrm{~d}$ \\
\hline Omissão de K & $137,1 \mathrm{c}$ & $74,1 \mathrm{~b}$ & $6,7 \mathrm{~d}$ & $10,2 \mathrm{e}$ & $16,9 \mathrm{e}$ & $6,2 \mathrm{~g}$ & $23,1 \mathrm{f}$ \\
\hline Omissão de Ca & $328,7 \mathrm{a}$ & 94,1 a & $11,9 \mathrm{a}$ & $24,3 \mathrm{~b}$ & $36,2 \mathrm{~b}$ & 7,7 ef & $43,9 \mathrm{c}$ \\
\hline Omissão de Mg & $331,6 \mathrm{a}$ & $90,6 \mathrm{a}$ & $11,5 \mathrm{ab}$ & $26,7 \mathrm{a}$ & $38,3 \mathrm{a}$ & $10,1 \mathrm{a}$ & $48,4 \mathrm{a}$ \\
\hline Omissão de $\mathrm{S}$ & $140,1 \mathrm{c}$ & $71,6 \mathrm{~b}$ & $9,5 \mathrm{c}$ & $14,4 \mathrm{~d}$ & $23,9 \mathrm{~d}$ & 8,3 de & $32,2 \mathrm{e}$ \\
\hline Omissão de micronutrientes & $334,5 \mathrm{a}$ & $91,5 \mathrm{a}$ & $11,9 \mathrm{a}$ & $26,7 \mathrm{a}$ & $38,6 \mathrm{a}$ & $8,5 \mathrm{~cd}$ & $47,1 \mathrm{ab}$ \\
\hline Controle & $29,8 \mathrm{~d}$ & $52,3 \mathrm{c}$ & $5,1 \mathrm{e}$ & $7,6 \mathrm{f}$ & $12,9 \mathrm{f}$ & $5,8 \mathrm{~g}$ & $18,6 \mathrm{~g}$ \\
\hline CV (\%) & 11,7 & 14,3 & 5,4 & 10,1 & 6,9 & 11,2 & 6,7 \\
\hline
\end{tabular}

* Tratamentos com médias não seguidas por mesma letra, na vertical, diferem pelo teste de Duncan a $5 \%$ de probabilidade de erro. 
nutriente. Contrariamente, as omissões de $\mathrm{Ca}, \mathrm{N}$ e $\mathrm{K}$ diminuíram consideravelmente a acumulação de matéria seca radicular. Além disso, a omissão de micronutrientes, que não apresentou efeito depressivo sobre a biomassa da parte aérea, manifestou-se, reduzindo a biomassa radicular. Levando-se em consideração que as raízes são os órgãos de interesse para uso na indústria farmacêutica, sugere-se a realização de estudos de longo prazo em nível de plantio a campo no teor dos fitofármacos-alvo. Esses estudos devem ter como base os resultados obtidos no presente trabalho (diminuição de produtividade de biomassa com a omissão de $\mathrm{Ca}, \mathrm{N}, \mathrm{P}, \mathrm{K}$ e S) e na experiência com outras culturas, como é o caso da necessidade de correção da acidez do solo, por se tratar de uma espécie cujo produto comercial é a raiz.

A produção de biomassa total da planta obedeceu a seguinte ordem decrescente, em relação à omissão do nutriente: $\mathrm{Mg} \cong$ micronutrientes $\geq$ adubação completa $\geq \mathrm{Ca}>\mathrm{P}>\mathrm{S}>\mathrm{K}>\mathrm{N} \cong$ controle (Tabela 2). A diferença de crescimento obtida pelos tratamentos de omissão de $\mathrm{Mg}$ e micronutrientes em relação ao controle e omissão de $\mathrm{N}$ foi de aproximadamente $145 \%$. A limitação do crescimento da $\boldsymbol{P}$. glomerata resultante da omissão de N, observada no presente trabalho e de outras espécies vegetais, é justificada em razão de esse nutriente ser o elemento mineral mais requerido pelas plantas (MARSCHNER, 2002) e também da insuficiente taxa de reposição do $\mathrm{N}$ mineral da solução via mineralização dos resíduos orgânicos e da matéria orgânica do solo. Para culturas que não formam associações com organismos fixadores de $\mathrm{N}$, elas dependem do $\mathrm{N}$ armazenado na matéria orgânica do solo, a qual é a fonte principal também de S e B. De acordo com os dados da tabela 1, observa-se que a porcentagem de matéria orgânica no Argissolo Vermelho distrófico arênico utilizado nesse trabalho é baixa (CQFS-RS/SC, 2004). O teor de sulfato no solo original é classificado como adequado às plantas em geral. No entanto, a omissão de enxofre provocou diminuição da sua biomassa, evidenciando que, ou o método de extração desse íon não é eficaz para o diagnóstico da sua real disponibilidade, ou esta espécie é muito exigente em enxofre.

O tratamento de adubação com omissão de K também afetou drasticamente o crescimento da $\boldsymbol{P}$. glomerata (Tabela 2), pois o teor disponível está abaixo do nível de suficiência estipulado para várias espécies pela CQFS-RS/SC (2004). Desse modo, a técnica do elemento faltante possibilitou comprovar que o Argissolo em estudo naturalmente não tem capacidade de fornecer K em taxas adequadas a P. glomerata. Por outro lado, essa técnica também permitiu, partindo-se de um valor de disponibilidade de $\mathrm{K}$ verificar que esta espécie tem comportamento similar as demais plantas já estudas, isto é, não produz adequadamente quando a classe de disponibilidade de $\mathrm{K}$ for média, baixa ou muito baixa. Tem-se observado que o nível de suficiência de K para culturas de grãos para esse solo é de aproximadamente $40 \mathrm{mg} \mathrm{dm}^{-3}$ e que a aplicação de baixas doses $\left(<60 \mathrm{~kg} \mathrm{~K}_{2} \mathrm{O}\right.$ ha $^{-1}$ ano $\left.^{-1}\right)$ já é suficiente para manter altas produtividades e o nível adequado de disponibilidade no solo (BRUNETTO et al., 2005). O mesmo comportamento foi observado para o $\mathrm{P}$, em que a sua disponibilidade natural é muito baixa (Tabela 1) (CQFS-RS/SC, 2004) e a probabilidade de respostas da ampla maioria das culturas é alta, como foi observado para a P. glomerata. Como o solo é arenoso e pobre em óxidos, a capacidade de adsorção de $\mathrm{P}$ é baixa, e consequentemente os teores de $\mathrm{P}$ disponíveis são facilmente elevados a valores muito altos com a adubação fosfatada (Tabela 1).

As respostas positivas no crescimento da P. glomerata às adubações contendo N, K, S e P são justificadas e, também, sugerem que esta espécie apresenta alto requerimento desses nutrientes para obter seu máximo crescimento e/ou, ainda, possui baixa eficiência de absorção desses nutrientes.

A classificação dos valores de $\mathrm{Ca}, \mathrm{Mg}$ e micronutrientes pela CQFS-RS/SC (2004) não tem a mesma fundamentação experimental do que para os nutrientes $\mathrm{N}, \mathrm{P}$ e $\mathrm{K}$ ou para a acidez do solo. A classificação é muito mais uma tentativa de organização dos resultados das análises de solo do que baseado na probabilidade de respostas das culturas (CQFS-RS/ $\mathrm{SC}, 2004)$. Desse modo, mesmo que os teores de $\mathrm{Mg}$ e $\mathrm{Ca}$ encontrados no solo natural sejam enquadrados na classe média ou baixa, as plantas de $\boldsymbol{P}$. glomerata já obtiveram o crescimento máximo, comportamento esse corriqueiramente observado para outras espécies. Adicionalmente, as interpretações referentes ao $\mathrm{Ca}$ devem ser vistas com cautela, pois sua disponibilidade efetiva depende da atividade de alumínio na solução do solo, que, no presente solo, deve ser alta.

Quanto aos micronutrientes no solo, independentemente dos tratamentos de adubação utilizados, os teores de $\mathrm{Cu}, \mathrm{Zn}, \mathrm{Fe}, \mathrm{Mn}$ e B são considerados altos (Tabela 1), segundo CQFS-RS/SC (2004). Portanto, esses dados sugerem que as disponibilidades desses elementos não são limitantes ao crescimento inicial das plantas de $\boldsymbol{P}$. glomerata cultivadas em Argissolo Vermelho distrófico arênico. Apenas em casos específicos, dependentes do tipo de solo e espécie vegetal, têm-se observado respostas positivas à aplicação de micronutrientes (LOPES \& ABREU, 2000).

O crescimento relativo $(\mathrm{CR})$ em produção de matéria seca da planta é mostrado na figura $1 \mathrm{~A}$. 


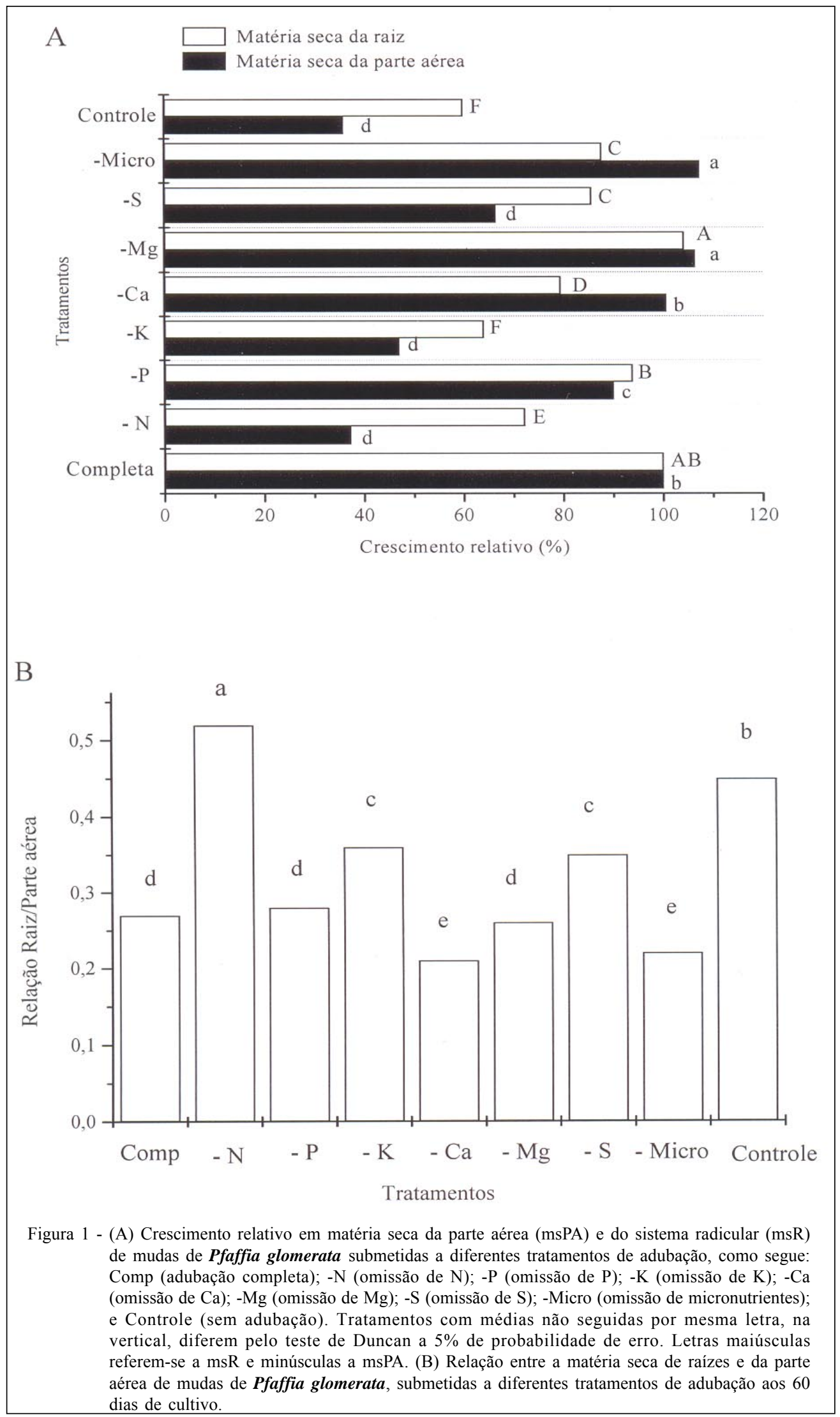

Ciência Rural, v.38, n.4, jul, 2008. 
Considerou-se o tratamento de adubação completa com CR igual a 100. Verifica-se que a maior alteração no crescimento relativo da parte aérea foi promovida pela omissão dos nutrientes N, K, S e P. De modo geral, a omissão de nutrientes da adubação afetou em menor proporção o crescimento em biomassa do sistema radicular do que o da parte aérea (Figura 1A). Os tratamentos com omissão de K, N, Ca, S, P e micronutrientes apresentaram alteração no $\mathrm{CR}$ da raiz em relação ao tratamento com adubação completa e de omissão de $\mathrm{Mg}$. Em plantas de angico-amarelo (Peltophorium dubium), as omissões de nutrientes da adubação que mais afetaram a produção de matéria seca da parte aérea foram de $\mathrm{P}, \mathrm{N}, \mathrm{S}$ e Ca (VENTURIN et al., 1999). VIÉGAS et al. (2004b), estudando a omissão de macronutrientes em camucamuzeiro (Myrciaria dubia) pela técnica do nutriente faltante em solução nutritiva, observaram que o crescimento relativo de toda a planta não foi afetado pela omissão de $\mathrm{P}$, foi menos afetado pela omissão de $\mathrm{Ca}$, com redução de $55 \%$ da matéria seca, e mais afetado pela omissão de N, com redução de $84 \%$ da matéria seca.

A partição de biomassa entre os órgãos da planta, analisada através da relação entre matéria seca das raízes e da parte aérea de mudas de $\boldsymbol{P}$. glomerata, foi principalmente afetada pelo tratamento de adubação com omissão de N, seguido do controle e da omissão de K e S (Figura 1B). É conhecido o fato de que o status nutricional da planta afeta o fracionamento de fotoassimilados, com reflexos na relação raiz/parte aérea (MARSCHNER, 2002; NICOLOSO et al., 2001b). Para o angico-amarelo (Peltophorium dubium), esta relação foi mais afetada no tratamento controle e naqueles com omissão de P, N e S (VENTURIN et al., 1999). De acordo com estes autores, a relação raiz/parte aérea é um bom parâmetro para avaliação da sobrevivência das mudas no campo. No entanto, esta relação é variável para cada espécie e condição ambiental utilizada no cultivo.

As caracterizações dos teores de macro e micronutrientes, bem como de $\beta$-ecdisona (principal substância utilizada como marcadora química em $\boldsymbol{P}$. glomerata) na matéria seca de raízes e parte aérea das plantas, obtidas no presente trabalho estão sendo realizadas. Isso permitirá uma primeira aproximação dos valores adequados de nutrientes para essa espécie e possibilitará traçar correlações relevantes entre as condições nutricionais e a acumulação de substâncias de interesse farmacológico.

\section{CONCLUSÕES}

Plantas jovens de $\boldsymbol{P}$. glomerata cultivadas em amostras de solo do horizonte superficial arenoso
(Argissolo Vermelho distrófico arênico) com baixos teores de matéria orgânica, baixo valor de $\mathrm{pH}$ e teores de fósforo e potássio disponíveis abaixo do teor crítico apresentaram grande diminuição no crescimento na ausência de adição de nitrogênio, potássio, enxofre e fósforo. Naturalmente, o solo forneceu adequadamente magnésio, boro, molibdênio, zinco, cobre, manganês e ferro às plantas de $\boldsymbol{P}$. glomerata. O comportamento dessa espécie, quanto à disponibilidade de nutrientes, é muito similar aos das demais espécies vegetais de interesse econômico estudadas até o presente momento.

\section{REFERÊNCIAS}

ASSIS, M.P. et al. Limitações nutricionais para a cultura do arroz em solos orgânicos sob inundação. Ciência e Agrotecnologia, Lavras, v.25, n.2, p.299-310, 2001.

BARROS, N.F.; NOVAIS, R.F. Relação solo-eucalipto. Viçosa: Folha de Viçosa, 1990. 330p

BATISTA, M.M.F. et al. Efeito da omissão de macronutrientes no crescimento, nos sintomas de deficiências nutricionais e na composição mineral em gravioleiras (Annona muricata). Revista Brasileira de Fruticultura, Jaboticabal, v. 25, n.2, p.315-318, 2003

BRUNETTO, G. et al. Nível crítico e resposta das culturas ao potássio em solo um solo de textura arenosa sob sistema plantio direto. Revista Brasileira de Ciência do Solo, Viçosa, v.29, n.4, p.565-571, 2005.

COMISSÃO DE QUÍMICA E FERTILIDADE DO SOLO - RS SC. Manual de adubação e calagem para os estados do Rio Grande do Sul e Santa Catarina. Porto Alegre: SBCSNRS, 2004. 394p.

FÖHSE, D. et al. Phosphorus efficiency of plants: I - external and internal $\mathrm{P}$ requirement and $\mathrm{P}$ uptake efficiency of different plant species. Plant and Soil, Dordrecht, v.110, p.101-109, 1988.

FREDEEN, A.L. et al. Influence of phosphorus nutrition on growth and carbon partitioning in Glycine max. Plant Physiology, Baltimore, v.89, p.225-230, 1989.

LOPES, A.S.; ABREU, C.A. Micronutrientes na agricultura brasileira: evolução histórica e futura. In: NOVAIS, R.F. et al. Tópicos em ciência do solo. Viçosa: Sociedade Brasileira de Ciência do Solo, 2000. V.1, p.265-298.

LYNCH, J. et al. Vegetative growth of the common bean in response to phosphorus nutrition. Crop Science, Madison, v.31, p.380-387, 1991 .

MAGALHAES, P.M. de. Agrotecnología para el cultivo de fáfia o ginseng brasilero. In: MARTINEZ, J.V. et al. Fundamentos de agrotecnología de cultivo de plantas medicinales iberoamericanas. Santafé de Bogotá: Convênio Andrés Bello/CYTED, 2000. p.323-332.

MALAVOLTA, E. Elementos de nutrição mineral de plantas. São Paulo: Ceres, 1980. 251p. 
MARSCHNER, H. Mineral nutrition of higher plants. London: Academic, 2002. 889p.

MARQUES, T.C.L.L.S. de. et al. Exigências nutricionais do Paricá (Schizolobium amazonicum,Herb.) na fase de muda. Revista Cerne, Lavras, v.10, n.2, p.167-183, 2004.

MONTANARI Jr., I. Aspectos do cultivo comercial do ginseng brasileiro (Pfaffia glomerata Spreng. Pedersen). São Paulo: CPQBA-UNICAMP, 1999. 3p. (Boletim Agroecológico, 12).

MONTANARI Jr., I. et al. Influence of plantation density and cultivation cycle on root productivity and tenors of $\beta$-ecdisone in Pfaffia glomerata (Spreng) Pedersen. Acta Horticulturae, Leuven, v.3, n.502, p.125-128, 1999.

MOREIRA, A. et al. Disponibilidade de nutrientes em vertissolo calcário. Pesquisa Agropecuária Brasileira, Brasília, v.35, n. 10, p.2107-2113, 2000.

MURASHIGE, T.; SKOOG, F. A revised medium for rapid growth and bioassays with tobacco tissue cultures. Physiologia Plantarum, Copenhagen. v.15, p.473-497, 1962.

NICOLOSO, F.T. et al. Exigências nutricionais da grápia (Apuleia leiocarpa Vog. Macbride) em solo podzólico vermelho amarelo. Ciência Rural, Santa Maria, v.29, n.2, p.225-231, 1999.

NICOLOSO, F.T. et al. Micropropagação do ginseng brasileiro [Pfaffia glomerata (Spreng.) Pedersen]. Revista Brasileira de Plantas Medicinais, Botucatu, v.3, n.2, p. 11-18, 2001a.
NICOLOSO, F.T. et al. Nutrição mineral de mudas de grápia (Apuleia leiocarpa Vog. Macbride) em Argissolo Vermelho distrófico arênico: (I) Efeito da adubação NPK no crescimento. Ciência Rural, Santa Maria, v.31, n.6, p.991-998, 2001b.

RENO, N.B. et al. Limitações nutricionais ao crescimento inicial de quatro espécies arbóreas nativas em latossolo vermelho-amarelo. Pesquisa Agropecuária Brasileira, Brasília, v.32, n.1, p.2071-2081, 1997.

SKREBSKY, E. C. et al. Substratos na aclimatização de Pfaffia glomerata (Spreng.) Pedersen produzida in vitro sob diferentes doses de sacarose. Ciência Rural, Santa Maria, v.36, n.5, p.1416-1423, 2006.

VIÉGAS, I.J.M. et al. Limitações nutricionais para o cultivo de açaizeiro em latossolo amarelo textura média, Estado do Pará. Revista Brasileira de Fruticultura, Jaboticabal, v.26, n.2, p.382-384, 2004a.

VIÉGAS, I.J.M. et al. Efeito da omissão de macronutrientes e boro no crescimento, nos sintomas de deficiências nutricionais e na composição mineral de plantas de camucamuzeiro. Revista Brasileira de Fruticultura, Jaboticabal, v.26, n.2, p.315-319, 2004b.

VENTURIN, N. et al. Adubação mineral do Angico-amarelo (Peltophorum dubium (Spreng.) Taub. Pesquisa Agropecuária Brasileira, Brasília, v.34, n.3, p.441-448, 1999. 